\title{
Automated Detection of White Blood Cells Cancer Diseases
}

\author{
T.Siva Prasanna, P.Jagadeesh
}

\begin{abstract}
Mechanized analysis of white platelets malignant growth infections, for example, Leukemia and Myeloma is a difficult biomed-ical inquire about point. Our methodology introduces out of the blue another best in class application that helps with diagnosing the white platelets infections. we break these sicknesses into two classifications, every classification contains like side effects infections that may confound in diagnosing. In light of the specialist's determination, one of two methodologies is actualized. Each methodology is connected on one of the two maladies classification by processing distinctive highlights. At last, Random Forest classifier is connected for ultimate choice. The proposed methodology means to early disclosure of white platelets malignancy, decrease the misdiagnosis cases notwithstanding improve the framework learning approach. In addition, permitting the specialists just to have the last tuning on the outcome acquired from the framework. The proposed methodology accomplished an exactness of $93 \%$ in the principal classification and $95 \%$ in the second class.
\end{abstract}

\section{Keywords: Matlab Software}

\section{INTRODUCTION}

The gore includes of a interruption of uncommon prison cell in a liquid named plasma. Gore includes of $55 \%$ plasma, and $45 \%$ by cells called formed mechanisms. The gore plays out a great agreement of imperative capacity By means for the hemoglobin controlled trendy the erythrocytes, it takes oxygen to the matters and folds the carbon dioxide (CO2). It equally passes on nutritious matters (for example amino acids, sugars, Mineral salts). Generally if there should be an occurrence of high seriousness illnesses where the death rates are more, the hanging tight time of patients for their reports, for example, blood test, MRI is more. The time taken for age of any of the test is from 1-7 days. In high hazard illnesses like Hepatitis B, it is suggested that the patient's holding up time should be as less as could be expected under the circumstances and the treatment ought to be begun right away. The present framework utilized by the pathologists for distinguishing proof of blood parameters is expensive and the time associated with age of the reports is likewise more now and then prompting loss of patient's life. Likewise the obsessive tests are costly, which are once in a while not reasonable by the patient. Henceforth, there ought to be a

Revised Manuscript Received on August 14, 2019.

T.Siva Prasanna, UG Scholar, Department of Electronics and Communication Engineering, Saveetha school of engineering, Saveetha Institute of Medical and Technical Sciences, Chennai, Tamil Nadu, India

.(Email: sivaprasanna.t@gmail.com)

Mr.P.Jagadeesh, Assistant Professor, Department of Electronics and Communication Engineering, Saveetha school of engineering, Saveetha Institute of Medical and Technical Sciences, Chennai, Tamil Nadu, India.

(Email:jagadeeshp@ saveetha.com) robotized framework where the blood reports ought to be produced in an exceptionally less time with as least expense as would be prudent. The proposed framework would be an activity to produce the blood test reports in least time and will be financially savvy. Right now the time taken by pathologist is 1-7 days to create the reports. The reports will get created after substance medications on blood tests which requires additional time. It is likewise expensive as the instruments utilized for recognizable proof of the blood parameters are exorbitant. The patients endure because of both these reasons physically just as rationally. In location process a few techniques utilized for the division of red platelet from white platelet Using shading space show with the assistance of MATLAB programming.

\section{OBJECTIVE}

In this work the strategy secludes and decides the sort of blood malignancy in the smear which could be either ordinary or strange and decides the nearness of blood white cell. Contemplating that irregular white platelets show to the related blood leukemia.

\section{EXISTING SYSTEM}

The paper entails an thought to build up a mechanized technique for examination of AML impact cell pictures and to incorporate into picture handling programming, which empowers the hematologist to analyze AML all the more adequately and efficiently.Haematologists frequently face troubles recognizing the subdivision of AML, because of the likenesses of their morphological highlights. Subsequent AML discovery, impact prison cell should remain neat addicted to M3 or unique of the different subtypes. The explanation behind focusing on M3 is that its action contrasts since the action of the rest, needful All-TransRetinoic-Acid (ATRA) to be extra to the underlying chemotherapy.

\section{PROPOSED METHOD}

The plan, improvement and evaluation of a computerized framework to precisely recognize platelets malignant growth maladies. It identifies types and sub-types of Leukemia (ALL and AML) and Myeloma. At that point performs preprocessing, division, highlight extractionand classification.In this work, the element extraction approach is executed which depends on a parametric model and made by a gathering out of scale invariants. Additionally, in this

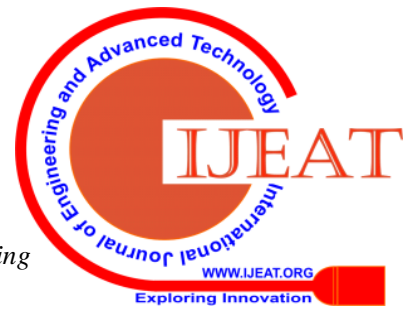


paper the order of blood smear pictures for leukemia discovery are appeared trial factual with a database smear platelet pictures. The sort of blood malignant growth in the smear which could be either typical or anomalous and decides the nearness of blood white cell. Mulling over that anomalous white platelets demonstrate to the related blood leukemia.

\section{RGB COLOR IMAGE}

The RGB covering model is an extra matter shading classical in which red, green, and blue light are incorporated into various ways to deal with reproduce a wide show of tones. The name of the model begins from the initials of the three included substance fundamental tones, red, green, and blue.

The essential inspiration driving the RGB shading model is for the recognizing, depiction, and show of pictures in electronic structures, for instance, TVs and PCs, anyway it has furthermore been used in customary photography. Preceding the electronic age, the RGB shading model recently had a solid theory behind it, arranged in human perspective on colors.RGB is a device subordinate shading model: assorted contraptions recognize or mimic a given RGB regard in a startling manner, since the shading parts, (for instance, phosphors or hues) and their response to the individual $\mathrm{R}, \mathrm{G}$, and $\mathrm{B}$ levels change from maker to producer, or even in a comparable device after some time. Thusly a RGB regard does not portray a comparative shading transversely over devices without some kind of shading the board. Run of the mill RGB input strategies are shading TV and camcorders, picture scanners, and automated cameras. Normal RGB yield contraptions are TV sets of various advances (CRT, LCD, plasma, etc.), PC and mobile phone appears, video projectors, multicolor LED exhibits, and immense screens, for instance, Jumbo Tron. Shading printers, on the other hand, are not RGB devices, yet subtractive shading devices .

\section{GRAYSCALE}

In taking pictures and figuring, a grayscale or greyscale electronic image is a image in which the estimate of every pixel is a solitary example, that is, it conveys just intensity information. Images of this sort, then called extremely conflicting, are complete solely out of tinted lenses of dim, changing from shady at the weakest control to white at the greatest ashore.

Grayscale images are specific from one-piece bi-tonal very conflicting images, which with respects to PC imaging are images with just the two types, shady, and white (likewise named bit level or parallel images). Grayscale images have frequent shades of dim in the central. Grayscale images are additionally called monochromatic, signifying the nearness of just one (mono) shading (chrome). Grayscale images are normally the consequence of approximating the power of bright at every pixel in a private group of the electromagnetic series (for example infrared, obvious bright,ultraviolet, and so on.), and in such suitcases they are monochromatic genuine when just a given reappearance is wedged. Yet in adding they can be combined from a full covering image; understand the part about altering ended to grayscaleExampleofgrayscaleimageisgivenbelow.

\section{MORPHOLOGICAL OPERATIONS \& RESULTS}

To locate the precise highlights we need to portion the lung district from the chest CT check picture for simple calculation. For fragmenting the lung district from the chest CT examine picture morphological task is completed. We characterized a picture as a (plentifulness) capacity of two, genuine (organize) factors $\mathrm{a}(\mathrm{x}, \mathrm{y})$ or binary, separate factors $\mathrm{a}[\mathrm{m}, \mathrm{n}]$. An elective meaning of a image can be founded on the supposed that a image includes of a usual (or gathering) of also nonstop or separate orders. One might say the set links to the attentions or pixels that have a residence with the items trendy the image. This is delineated in number beneath which contains two exercises or sets An and B. Memo that the arrange outline is obligatory. For the minute we will consider the pixel esteems to be paired as examined in the Further we will confine our talk to discrete space.

\section{Steps in image processing}

\section{Preprocessing}

The reason for the pre-handling stage is to expel undesirable impacts, for example, clamor from the picture, and change or modify the picture as essential for further preparing. The goals of the picture is diminished by a factor of four to $512 \cdot 384$ to accelerate execution of the framework. Likewise, the test pictures will be exposed to specific middle separating and unsharp covering to segregate clamor which may have been aggregated amid picture securing and because of over the top recoloring.

\section{Segmentation}

The strategy of apportioning the picture into portion can be characterized as picture division. Considering the comparative property, segmentation is executed. This comparative property is bunch together our propounded methodology actualizes Lloyd's clustering technique which helps in the division of blood minute pictures based on alike properties. This method broadens the k-mean bunching calculation by presenting rehashed division conspire which investigates the centroid of each set in the segment and in the long run re-portion the info dependent on the nearest centroid. This method helps in the extraction of important image attributes, in light of which data can be effectively perceived.A basic thresholding method stands connected to stretch beginning names to pixels trendy the platelet pictures. The calculation depends on apriori data about blood smear pictures. At that point the marks are balanced with a shape discovery technique dependent on vast regional context data to deliver significant outcomes.

\section{K-Mean Clustering}

Isolating of the data space into Voronoi cells. The issue is computationally irksome (NP-hard); nevertheless, capable heuristic figuring join quickly to an area perfect. These are normally similar to the longing expansion computation for 
mixes of Gaussian scatterings through an iterative refinement approach used by both k-infers and Gaussian mix illustrating. The two of them use bundle centers to demonstrate the data; regardless, k-suggests gathering will by and large find gatherings of essentially indistinguishable spatial degree, while the longing increase instrument empowers packs to have differing shapes. The computation has a free relationship to the k-nearest neighbor classifier, a standard AI strategy for gathering that is routinely confused with k-infers on account of the name. Applying the 1nearest neighbor classifier to the gathering centers gotten by $\mathrm{k}$-infers organizes new data into the present bundles. This is known as nearest centroid classifier figuring.

\section{Feature Extraction}

In sample acknowledgement and in image preparing, contain extractionis an uncommon kind of dimensionality decrease. At the opinion when the info data to a calculation is also huge to ever be touched then it is supposed to be well extra, at that point the information data determination be altered addicted to a decreased portrayal usual of highlights. Changing the information data into the preparation of highlights is named include removal. On the off chance that the places of interest removed are purposely selected it is usual that the places of interest usual will extricate the important information after the info information so as to production available the ideal undertaking using this decreased depiction somewhat than the full scope info. Highpoint removal comprises rearranging the amount of possessions obligatory to depict a vast preparation of info exactly. Once execution investigation of multifaceted info unique of the serious subjects comes since the amount of issues comprised. Study by a substantial amount of features by and big needs a share of recall then control change or a characterization control which over fits the training exam and calculations up vainly to new-fangled samples. Highlight removal is a overall period aimed at strategies used for developing mixes of the issues to become about these matters though as up till now depicting the data with passable care.

\section{KNN Classification}

The KNN two fold (as two class) is given progressively exact information characterization which advantageous to choose $\mathrm{k}$ as an odd number which avoids the unpredictable information. The KNN strategy is the method in ML strategies: It is an item which grouped through a main stream choice of its neighbors, with the assurance doled out event for most shared session among its $\mathrm{k}$ adjacent neighbors ( $\mathrm{k}$ is a confident number, traditionally little). Traditionally Euclidean separation is utilized as the separation metric; nonetheless, this is only reasonable for unlimited factors. $\mathrm{KNN}$ is another procedure that conveyances every single accessible case and arranges novel cases based on an assessment amount (e.g., detachment functions).KNN method is indistinguishable straightforward. It works based on a base separation from the cross examination occasion to the preparation simplest control the K-closest neighbors. The data for KNN technique contains various trait which will be utilized to catalogue. The data of KNN can be any measurement scale from irrelevant, to quantifiable scale.

\section{CONCLUSION}

In this paper we suggest the structure, improvement besides assessment of a mechanized framework to precisely distinguish white platelets malignant growth infections. It identifies types and sub-sorts of Leukemia (ALL and AML) and Myeloma. It is a recogni-tion framework connected on gained blood infinitesimal pictures at that point performs preprocessing, division, include extraction and order. The proposed arrangement changes over pictures to YCBCR shading space and build Gaussian circulation of $\mathrm{CB}$ and $\mathrm{CR}$ esteems. Measurable, surface, estimate proportion and morpho-consistent highlights are then processed to prepare classifier. In contrast to existing frameworks, our framework has the capacity of gaining from misclassified tests to improve the future precision of the framework. Irregular Forest classifier is the best classifier that can separate between various sorts and the one which gives us the best precision. The framework accomplished $94.3 \%$ exactness in identifying and ordering types and sub-types. As our following stage, we mean to recognize more types of white platelets cancer diseases to fabricate an overall system for white platelets illnesses.

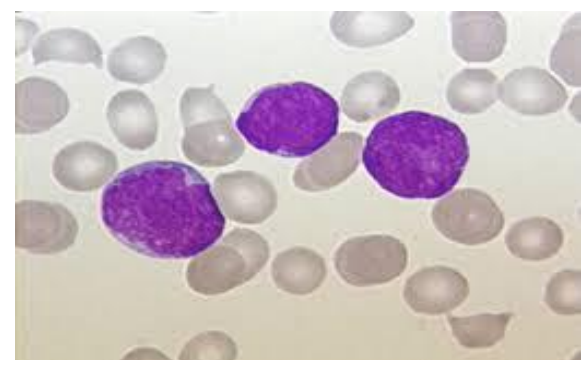

Fig 1. Input Image

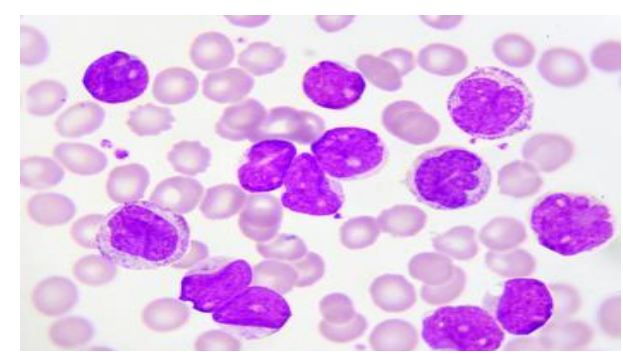

Fig 2. Estimated Image

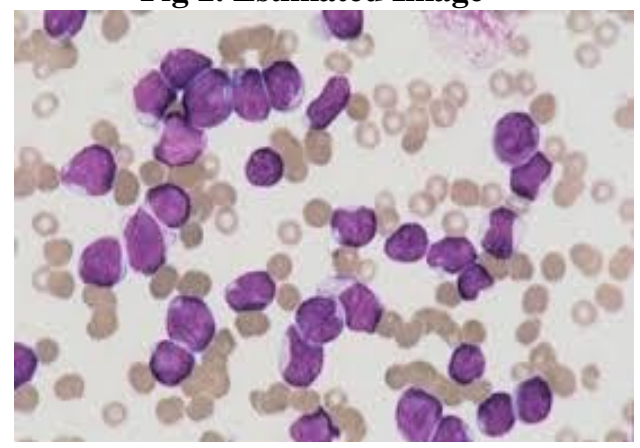

Fig 3. ALL 


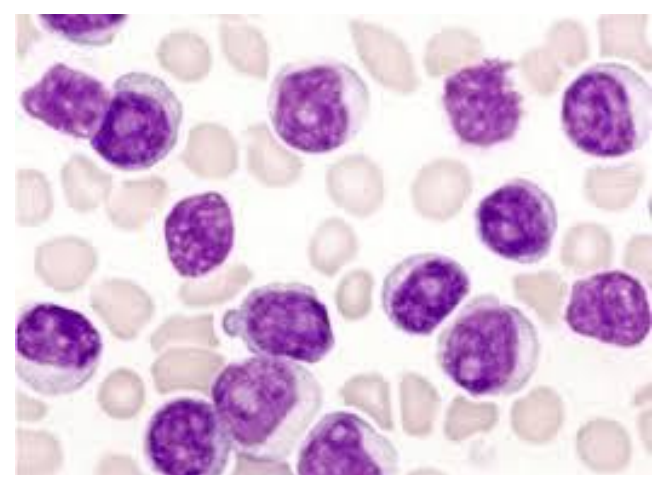

Fig 4.AML

\section{REFERENCES}

1. Cristianini, N., and J. Shawe-Taylor. "An Introduction to support vector machines and other kernel-based learning methods" New York: Cambridge University Press, 2000.

2. Vapnik, V. N. "The Ature of Statistical Learning Theory" New York: Springer, 1995.

3. A. Madabhushi, "Digital pathology imageanalysis:opportunitiesandchallenges," Imaging in Medicine, vol. 1, no. 1, pp. 7-10, 2009.

4. A. N. Esgiar, R. N. G. Naguib, B. S. Sharif, M. K. Bennett, and A. Murray, "Fractal analysis in the detection of colonic cancer images," IEEE Transactions on Information Technology in Biomedicine, vol. 6, no. 1, pp. 54-58, 2002.

5. L. Yang, O. Tuzel, P. Meer, and D. J. Foran, "Automatic image analysis of histopathology specimens using concave vertex graph," in Medical Image Computing and Computer-Assisted Intervention-MICCAI 2008, pp. 833-841, Springer, Berlin, Germany, 2008.

6. R. C. Gonzalez, Digital Image Processing, Pearson Education India, 2009.

7. S. Liao, M. W. K. Law, and A. C. S. Chung, "Dominant local binary patterns for texture classification," IEEE Transactions on Image Processing, vol. 18, no. 5, pp. 1107-1118, 2009.

8. J. C. Caicedo, A. Cruz, and F. A. Gonzalez, "Histopathology image classification using a bag of features and kernel functions," in Artificial Intelligence in Medicine, vol. 5651 of Lecture Notes in Computer Science, pp. 126-135, Springer, Berlin, Germany, 2009.

9. H. S. Wu, J. Barba, and J. Gil, "Iterative thresholding for segmentation of cells from noisy images" Journal of Microscopy, vol. 197, no. 3, pp. 296-304, 2000.

10. C. Di Rubeto, A. Dempster, S. Khan, and B. Jarra, "Segmentation of blood images using morphological operators," in Proceedings of the 15th International Conference on Pattern Recognition, vol. 3, pp. 397-400, 2000.

11. C.D., Ruberto, A., Dempster, S., Khan, B. Jarra, "Analysis of Infected Blood Cell Images Using Morphological Operators", Image and Vision Computing, Vol. 20, 2002. 